\title{
Determinação de efedrinas em urina por cromatografia em fase gasosa (CG/DNP) para o controle da dopagem no esporte
}

\author{
Paula Rodrigues Garcia, Mauricio Yonamine*, Regina Lúcia de Moraes Moreau
}

Laboratório de Análises Toxicológicas, Departamento de Análises Clínicas e Toxicológicas, Faculdade de Ciências Farmacêuticas, Universidade de São Paulo

*Correspondência

Mauricio Yonamine

Laboratório de Análises Toxicológicas Faculdade de Ciências Farmacêuticas - USP

Av. Prof. Lineu Prestes, 580 B13B 05508-900 -São Paulo - Brasil

E-mail: yonamine@usp.br
Efedrinas são aminas simpatomiméticas componentes de diversas especialidades farmacêuticas, utilizadas no tratamento de doenças respiratórias devido à sua ação descongestionante e broncodilatora. Atualmente, diversos produtos comercializados como suplementos nutricionais contêm efedrinas e são amplamente utilizados no meio esportivo, com o objetivo de facilitar a queima de gorduras e melhorar o desempenho. Entretanto, o uso indiscriminado destas substâncias pode acarretar série de efeitos tóxicos como hipertensão, taquicardia, cefaléia e tremores. Devido à sua ação psicoestimulante, foram incluídas na lista de substâncias proibidas nas atividades esportivas pelo Comitê Olímpico Internacional (COI) e estabelecidas concentrações na urina para o controle da dopagem (efedrina e metilefedrina: $10 \mu \mathrm{g} / \mathrm{mL}$ ). O presente trabalho teve como objetivo a validação de um método para quantificação de efedrinas, por cromatografia em fase gasosa acoplada a detetor de nitrogênio/fósforo (CG/ $D N P$ ), em amostras de urina com a finalidade de controle da dopagem. O método consistiu em extração líquido-líquido e posterior derivação das efedrinas com anidrido trifluoroacético, e demonstrou ser simples e prático, apresentando linearidade nas faixas de concentração estudadas. Amostras de urina de voluntários que relataram uso de efedrinas foram submetidas à análise pelo método proposto.

\section{INTRODUÇÃO}

Efedrinas são agonistas simpatomiméticos de ação mista e, devido a seus efeitos em receptores $\beta 2$-adrenérgicos, são usadas na terapêutica no tratamento de gripes, resfriados e asma. A fenilpropanolamina, muito utilizada em formulações de descongestionantes nasais, foi retirada
Unitermos

- Efedrinas

- Dopagem no esporte

- Cromatografia em fase gasosa do mercado brasileiro pela ANVISA (Agência Nacional de Vigilância Sanitária), à vista da correlação de seu uso com casos de acidente vascular encefálico (Brasil, 2000). As efedrinas estão presentes, também, na composição de diversos suplementos nutricionais, na forma de Ma-Huang (Ephedra sp) sua fonte natural. Alcalóides da Ephedra incluem: efedrina $(\mathrm{EPH})$, pseudoefedrina $(\mathrm{PEPH})$, 
metilefedrina (MEPH), norefedrina (NEPH) (fenilpropanolamina), norpseudoefedrina (catina) e metilpseudoefedrina (Vansal, Feller, 1999; Juhn, 2003; Lieberman, 2001; Berlin, Warot, Aymard, 2001; Bohn, Khodaee, Schwenk, 2003; Bent et al., 2003).

O mercado de produtos vendidos como suplementos nutricionais cresceu consideravelmente a partir da metade da década de 1990. Um forte indicativo desta tendência, pode ser observado no aumento da venda destes produtos nos Estados Unidos: de 8,3 bilhões de dólares, em 1994, para 14 bilhões, em 1999 (Gurley, Gardner, Hubbarbd, 2000; Delbeke et al., 2003; Yonamine, Garcia, Moreau, 2004). A crença de que este tipo de suplemento é natural e que, portanto, não causa prejuízos à saúde, contribui para o aumento da popularidade dos produtos.

Suplementos nutricionais contendo Ma-Huang são comercializados principalmente para programas de emagrecimento e aumento do desempenho esportivo (Bent $e t$ al., 2003). Nestas circunstâncias, questões quanto à segurança, efeitos adversos e eficácia têm sido levantadas. Estudo realizado por Bent et al. (2003) demonstrou que, apesar destes suplementos representarem $0,82 \%$ das vendas de produtos naturais nos Estados Unidos, são responsáveis por $64 \%$ dos relatos de efeitos adversos associados ao uso de plantas medicinais.

Food and Drug Administration (FDA) recebeu, até 1997 , mais de 800 relatos de efeitos adversos associados ao consumo de produtos contendo alcalóides da Ephedra, em geral relacionados ao sistema nervoso central (SNC) e cardiovascular, incluindo hipertensão, arritmias, infarto do miocárdio, acidente vascular encefálico, cefaléia, convulsão, resultando em lesões permanentes e alguns casos fatais (Lieberman, 2001). Estes efeitos podem ser intensificados durante exercícios físicos e quando associados a outros fármacos estimulantes do SNC, como a cafeína (Vansal, Feller, 1999; Lieberman, 2001; Berlin, Warot, Aymard, 2001; Juhn, 2003).

Os efeitos estimulantes no SNC das efedrinas e a ampla disponibilidade no mercado levaram à sua utilização, no meio esportivo, como alternativa para os agentes anfetamínicos, fato este evidente na década de 1970 (Catlin, Murray, 1996). Como forma de controlar o abuso em atividades esportivas, o COI (Comitê Olímpico Internacional) classificou as efedrinas como substâncias proibidas e estabeleceu limites de concentração urinária para o controle da dopagem (para efedrina e metilefedrina: $10 \mu \mathrm{g} / \mathrm{mL}$ e para pseudoefedrina e norefedrina: $25 \mu \mathrm{g} / \mathrm{mL}$ ), que vigoraram até o ano de 2003 (International Olympic Committee, 2003). A partir de janeiro de 2004, uma nova lista foi divulgada pelo COI, no qual a pseudoefedrina e a norefedrina deixaram de ser substâncias banidas, com a ressalva de que seu uso continuasse a ser monitorado no esporte (International Olympic Committee, 2004). Portanto, o controle da utilização destes fármacos no esporte é necessário para prevenção dos efeitos relacionados ao seu uso e garantir a igualdade competitiva.

Desta forma, a finalidade do presente trabalho foi o desenvolvimento de um método analítico para quantificação de efedrina (EPH), pseudoefedrina (PEPH), norefedrina (NEPH) e metilefedrina (MEPH) em urina, utilizando extração líquido-líquido e a técnica de cromatografia em fase gasosa acoplada a detetor de nitrogênio/fósforo (CG/DNP), para ser aplicado a programas de controle da dopagem no esporte.

\section{MATERIAL E MÉTODOS}

\section{Soluções-padrão}

Soluções-padrão de 3,4-metilenodioximetanfetamina (MDMA), utilizada como padrão interno, EPH, PEPH e NEPH nas concentrações de $1 \mathrm{mg} / \mathrm{mL}$ em metanol foram obtidas da Radian International (Austin, EUA). MEPH (1 g) foi obtida da Sigma (St Louis, EUA). As soluções foram diluídas em metanol, de acordo com as necessidades analíticas e armazenadas $\mathrm{a}-20^{\circ} \mathrm{C}$.

\section{Solventes e Reagentes}

Os solventes éter terc-butilmetílico, metanol e acetato de etila foram obtidos da Merck (Darmstadt, Germany), assim como cloreto de sódio, hidróxido de potássio e sulfato de sódio anidro, utilizados no processo de extração. $\mathrm{O}$ anidrido trifluoroacético (ATFA) foi adquirido da Aldrich (Milwaukee, EUA).

\section{Amostras de referência negativa}

Para a validação do método foram empregadas amostras de urina de referência negativa, obtidas de voluntários que não fizeram uso de produtos ou medicamentos contendo os fármacos analisados.

\section{Amostras de controle de qualidade}

Amostras de referência negativa adicionadas com padrões de efedrinas nas seguintes concentrações: 1,0; 6,0 e $20,0 \mu \mathrm{g} / \mathrm{mL}$ para efedrina e metilefedrina e 2,0; 15,0 e $35,0 \mu \mathrm{g} / \mathrm{mL}$ para pseudoefedrina e norefedrina foram utilizadas como controle de qualidade para níveis baixo, médio e alto, respectivamente. 


\section{Amostras obtidas de voluntários}

Amostras de urina foram obtidas de voluntários (homens ou mulheres) que fizeram uso de suplementos nutricionais ou medicamentos contendo as efedrinas em estudo. A coleta das amostras foi realizada de acordo com o projeto de pesquisa aprovado pelo Comitê de Ética e Pesquisa da Faculdade de Ciências Farmacêuticas da Universidade de São Paulo (Ofício CEP nº 103/2002).

\section{Cromatografia em fase gasosa}

Foi utilizado equipamento de cromatografia em fase gasosa da Hewlett-Packard 6890N (Little Falls, EUA) equipado com detector de nitrogênio/fósforo (CG/DNP) nas seguintes condições: injeção split (1:20); coluna capilar de sílica fundida 5\% fenilmetilsilicone com as dimensões de $25 \mathrm{~m}$ x 0,2 mm x 0,33 $\mu \mathrm{m}$ (HP-Ultra 2). As temperaturas do injetor e detector foram $270{ }^{\circ} \mathrm{C} \mathrm{e} 325^{\circ} \mathrm{C}$, respectivamente; nitrogênio foi usado como gás de arraste a um fluxo constante de $0,4 \mathrm{~mL} / \mathrm{min}$; programação da temperatura do forno: $148{ }^{\circ} \mathrm{C}(1 \mathrm{~min}), 5^{\circ} \mathrm{C} / \mathrm{min}$ até $185^{\circ} \mathrm{C}$, $25^{\circ} \mathrm{C} / \mathrm{min}$ até $270{ }^{\circ} \mathrm{C}(4 \mathrm{~min})$. O equipamento foi operado pelo GC Chemstation, programa responsável pela integração e processamento dos cromatogramas.

\section{Preparo das amostras}

Alíquotas de $0,2 \mathrm{~mL}$ das amostras de urina a serem analisadas foram alcalinizadas com $0,02 \mathrm{~mL}$ de $\mathrm{KOH} 5 \mathrm{M}$. Em seguida, foram adicionados $20 \mathrm{mg}$ de $\mathrm{NaCl}$ e o padrão interno $(0,02 \mathrm{~mL}$ da solução-padrão de MDMA na concentração de $0,1 \mathrm{mg} / \mathrm{mL}$ ). Os analitos foram extraídos com 2,5 $\mathrm{mL}$ de éter terc-butilmetílico, após agitação por 10 min e centrifugação a $300 \mathrm{~g}$ por $5 \mathrm{~min}$. A fase orgânica foi transferida para um béquer contendo aproximadamente $200 \mathrm{mg}$ de sulfato de sódio anidro. O extrato foi transferido para um tubo de derivação e o solvente evaporado sob fluxo de nitrogênio à temperatura ambiente. $O$ resíduo foi submetido a reação de derivação adicionando-se $50 \mu \mathrm{L}$ de anidrido trifluoroacético (ATFA) e $50 \mu \mathrm{L}$ de acetato de etila, mantendo-se a $70^{\circ} \mathrm{C}$ por 15 minutos. Após a derivação, o excesso de reagente foi evaporado sob fluxo de nitrogênio e o resíduo reconstituído com $50 \mu \mathrm{L}$ de acetato de etila. Após uma hora de repouso, $2 \mu \mathrm{L}$ foram injetados no equipamento de cromatografia em fase gasosa (CG/DNP).

\section{Validação do método}

A validação do método foi realizada com o estabelecimento de valores de limite de detecção, limites de quantificação, linearidade, recuperação, precisão intra e interensaio e especificidade para as efedrinas, conforme descrito abaixo:

\section{Limites de detecção e quantificação}

Os limites de detecção (LD) e de quantificação (LQ) para cada analito foram obtidos pelo método empírico, que consiste na análise de uma série de amostras de urina de referência negativa, adicionadas com as soluçõespadrão das efedrinas em concentrações decrescentes. $\mathrm{O}$ limite de detecção foi considerado a menor concentração $(\mu \mathrm{g} / \mathrm{mL}$ de urina) que apresentou coeficiente de variação que não excedeu $20 \%$ nas análises de seis replicatas. O limite de quantificação foi considerado a menor concentração $(\mu \mathrm{g} / \mathrm{mL}$ de urina) que apresentou coeficiente de variação abaixo de $10 \%$ nas análises de seis replicatas (Chasin et al., 1998; Armbruster et al., 1994; Needleman, Romberg, 1990).

\section{Recuperação}

A medida da recuperação de cada analito foi estabelecida para as concentrações correspondentes aos controles de qualidade baixo, médio e alto.

A recuperação foi determinada pela relação das respostas obtidas quando as soluções-padrão das efedrinas foram adicionadas antes da extração com as respostas das amostras adicionadas após a extração em seis replicatas. O padrão interno (MDMA) foi adicionado antes da extração nos dois conjuntos de amostras.

\section{Linearidade}

O estudo de linearidade foi realizado pela análise de amostras de urina em triplicata, submetidas ao método proposto nas seguintes concentrações: 0,$3 ; 2,0 ; 4,0 ; 8,0 ; 15,0$ e $25,0 \mu \mathrm{g} / \mathrm{mL}$ (para efedrina), 0,$5 ; 2,0 ; 4,0 ; 8,0 ; 15,0$ e $25,0 \mu \mathrm{g} / \mathrm{mL}$ (para metilefedrina), 0,$3 ; 5,0 ; 10,0 ; 20,0 ; 30,0$ e $40,0 \mu \mathrm{g} / \mathrm{mL}$ (para norefedrina e pseudoefedrina). O coeficiente de correlação linear $\left(\mathrm{r}^{2}\right)$ foi calculado para cada curva de calibração.

\section{Medidas de precisão intra e interensaio}

As medidas de precisão intra e interensaios foram realizadas analisando-se amostras de controle de qualidade, conduzidas em sextuplicata por três dias. Os valores foram expressos através dos coeficientes de variação do método $(\mathrm{CV})$. 


\section{Especificidade}

A especificidade do método proposto foi verificada analisando-se amostras de urina de referência negativa adicionadas de outras aminas simpatomiméticas e demais fármacos presentes em especialidades farmacêuticas contendo as efedrinas: anfetamina, metanfetamina, 3,4metilenodioxianfetamina (MDA), 3,4-metilenodioxietilanfetamina (MDEA), cafeína, femproporex, aminofilina, clorfeniramina e difenidramina.

\section{RESULTADOS}

A Figura 1 apresenta um cromatograma de uma amostra de referência negativa adicionada com o padrão interno (MDMA) e um cromatograma de uma amostra de referência negativa adicionada dos padrões de efedrina, norefedrina, metilefedrina e pseudoefedrina nas concentrações relativas ao controle de qualidade médio. Na Figura 2 é mostrado o perfil cromatográfico obtido com a análise de uma amostra de urina de voluntário contendo $14,86 \mu \mathrm{g} / \mathrm{mL}$ de efedrina; $3,31 \mu \mathrm{g} / \mathrm{mL}$ de norefedrina e $0,87 \mu \mathrm{g} / \mathrm{mL}$ de pseudoefedrina.

As curvas de calibração foram lineares nas faixas de concentração estudadas para os fármacos analisados: efedrina (0,3 a $25 \mu \mathrm{g} / \mathrm{mL})$, metilefedrina $(0,5$ a $25 \mu \mathrm{g} /$ $\mathrm{mL})$; pseudoefedrina e norefedrina $(0,3 \mathrm{a} 40 \mu \mathrm{g} / \mathrm{mL})$. As equações de regressão linear e os coeficientes de correlação foram: $\mathrm{Y}=0,0324 \mathrm{X}+0,0672, \mathrm{r}^{2}=0,993$ (norefedrina); $\mathrm{Y}=0,0334 \mathrm{X}+0,031, \mathrm{r}^{2}=0,9914$ (efedrina); $\mathrm{Y}=0,0518 \mathrm{X}+0,0598, \mathrm{r}^{2}=0,9902$ (pseudoefedrina); $\mathrm{Y}=0,0608 \mathrm{X}-0,0572, \mathrm{r}^{2}=0,9913$ (metilefedrina), onde $\mathrm{Y}$ representa a área relativa (relação entre a área do pico produzido pelo analito e a área do pico produzido pelo padrão interno) e X corresponde às concentrações dos analitos.

Os parâmetros de validação do método estudado (limite de detecção, limite de quantificação, recuperação, precisão intra e interensaios) para determinação de efedrina, norefedrina, pseudoefedrina e metilefedrina são apresentados na Tabela I.

Com relação ao estudo de especificidade os resultados dos tempos de retenção relativos ao padrão interno são apresentados na Tabela II.

Amostras de urina de voluntários que relataram uso de suplementos nutricionais e/ou medicamentos contendo efedrinas foram submetidas ao método proposto. Os resultados das análises e os dados obtidos através da aplicação de questionários estão na Tabela III.

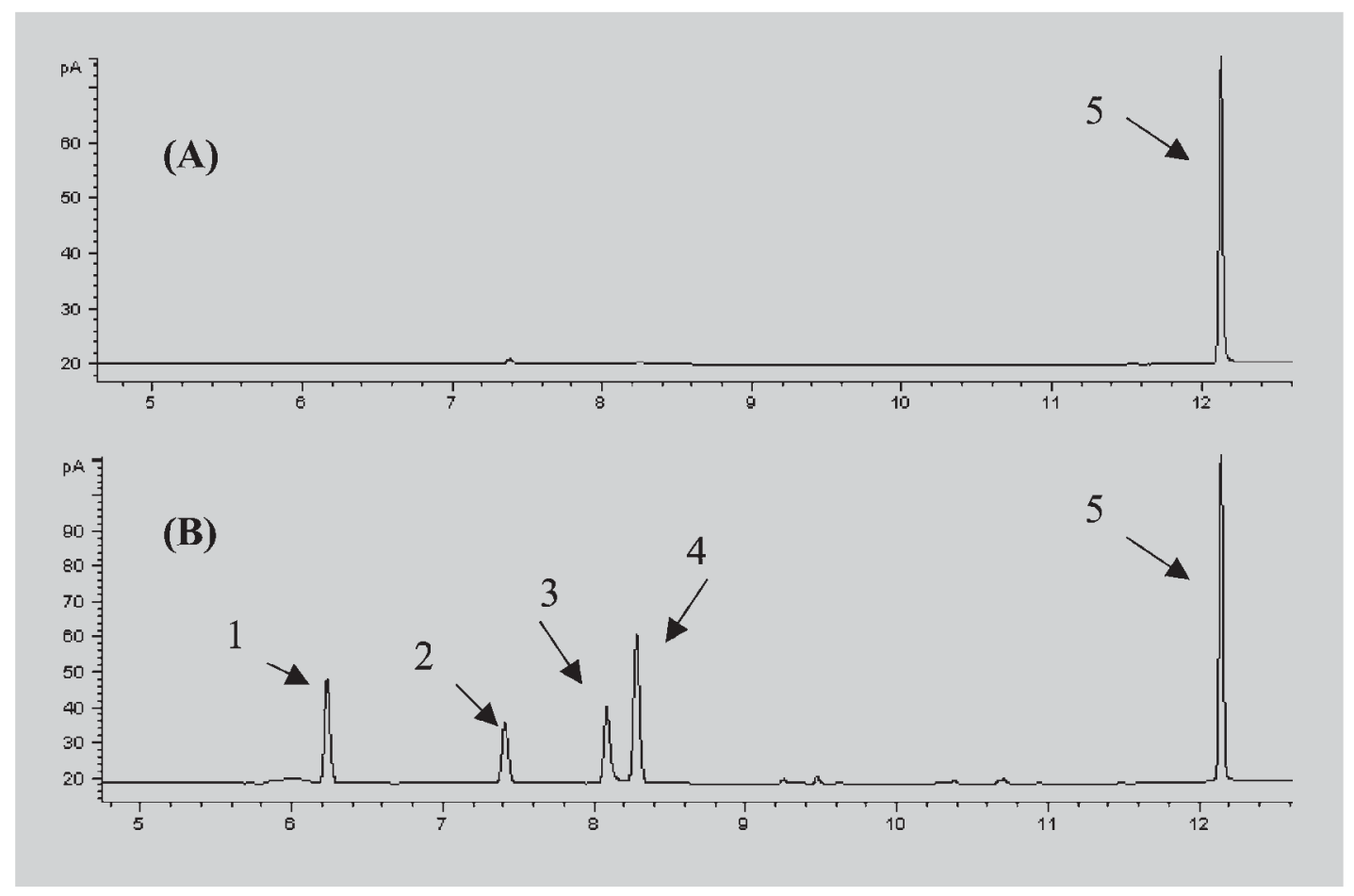

FIGURA 1 - (A) Perfil cromatográfico obtido com a análise de amostra de referência negativa. (B) Perfil cromatográfico obtido com a análise de uma amostra de urina de referência negativa adicionada com $6 \mu \mathrm{g} / \mathrm{mL}$ de efedrina (2) e metilefedrina (3) e $15 \mu \mathrm{g} / \mathrm{mL}$ de norefedrina (1) e pseudoefedrina (4). Padrão interno = MDMA (5). 


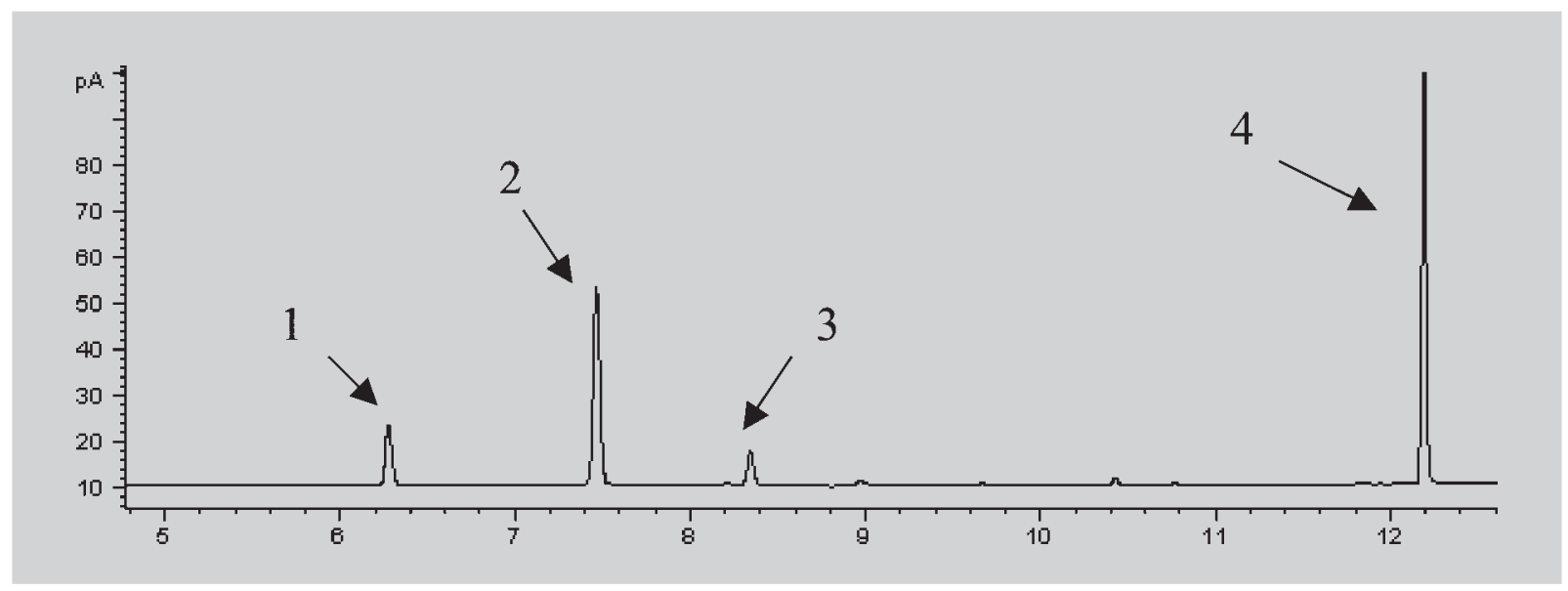

FIGURA 2 - Perfil cromatográfico obtido com a análise de uma amostra de urina de voluntário contendo 3,31 $\mu \mathrm{g} / \mathrm{mL}$ de norefedrina (1); $14,86 \mu \mathrm{g} / \mathrm{mL}$ de efedrina (2) e $0,87 \mu \mathrm{g} / \mathrm{mL}$ de pseudoefedrina (3). Padrão interno = MDMA (4).

TABELA I - Parâmetros de validação do método estudado para determinação de efedrina, norefedrina, pseudoefedrina e metilefedrina

\begin{tabular}{lcccc}
\hline & EPH & NEPH & PEPH & MEPH \\
\hline Limite de Detecção $(\mu \mathrm{g} / \mathrm{mL})$ & 0,1 & 0,1 & 0,1 & 0,3 \\
& $(\mathrm{CV})(15,9)$ & $(16,7)$ & $(15,9)$ & $(12,4)$ \\
Limite de Quantificação $(\mu \mathrm{g} / \mathrm{mL})(\mathrm{CV})$ & 0,3 & 0,3 & 0,3 & 0,5 \\
& $(10,0)$ & $(5,6)$ & $(6,1)$ & $(8,6)$ \\
Recuperação (\%) & & & & \\
CQ baixo $(\mathrm{CV})$ & $85,4(8,8)$ & $79,2(4,2)$ & $75,0(4,4)$ & $89,8(7,4)$ \\
CQ médio $(\mathrm{CV})$ & $67,1(7,9)$ & $75,6(5,5)$ & $76,8(5,0)$ & $81,4(6,0)$ \\
CQ alto $(\mathrm{CV})$ & $82,2(5,5)$ & $81,8(6,4)$ & $85,9(6,4)$ & $86,6(6,4)$ \\
Precisão intra-ensaio $(\mathrm{CV})$ & & & & \\
CQ baixo & 7,0 & 7,9 & 8,5 & 6,8 \\
CQ médio & 5,4 & 6,3 & 6,3 & 8,8 \\
CQ alto & 5,8 & 4,5 & 5,9 & 8,5 \\
Precisão inter-ensaio $(\mathrm{CV})$ & & & & \\
CQ baixo & 7,2 & 10,0 & 14,8 & 17,6 \\
CQ médio & 2,7 & 10,0 & 3,3 & 5,7 \\
CQ alto & 13,4 & 7,8 & 2,6 & 15,9 \\
\hline
\end{tabular}

$\mathrm{EPH}=$ efedrina; $\mathrm{NEPH}=$ norefedrina $\mathrm{PEPH}=$ pseudoefedrina; $\mathrm{MEPH}=$ metilefedrina; $\mathrm{CQ}=$ controle de qualidade; $\mathrm{CQ}$ = baixo, médio e alto para EPH e MEPH: 1, 6 e $20 \mu \mathrm{g} / \mathrm{mL}$; para NEPH e PEPH: 2, 15 e $35 \mu \mathrm{g} / \mathrm{mL}$; CV - coeficiente de variação.

\section{DISCUSSÃO}

Nos últimos anos, em virtude da grande exposição na mídia, da supervalorização de atletas e da extensa comercialização do esporte, a utilização de substâncias ou métodos físicos têm se tornado prática comum entre esportistas que buscam melhorar, artificialmente, seu desempenho numa determinada atividade física. Essa prática, conceituada como dopagem, é a antítese dos fundamentos que re- gem a filosofia desportiva como disciplina, dedicação, lealdade e competitividade (Silva, Yonamine, 2004).

Além de ser uma transgressão ética, a dopagem representa um risco para a saúde de quem a utiliza, pois é grande a probabilidade de efeitos tóxicos quando atletas utilizam substâncias em doses excessivas, sem qualquer respaldo científico.

Dentre as classes de substâncias proibidas no esporte, os estimulantes do SNC representam uma parcela sig- 
TABELA II - Tempos de retenção absolutos e relativos ao MDMA (padrão interno) obtidos para as substâncias pesquisadas no estudo de especificidade do método

\begin{tabular}{lcc}
\hline Substância & Tempo de retenção absoluto (min) & Tempo de retenção relativo \\
\hline Anfetamina & 6,12 & 0,502 \\
Norefedrina & 6,23 & 0,513 \\
Efedrina & 7,41 & 0,610 \\
Metanfetamina & 7,92 & 0,649 \\
Metilefedrina & 8,07 & 0,664 \\
Pseudoefedrina & 8,29 & 0,682 \\
Aminofilina & 10,35 & 0,848 \\
3,4-Metilenodioxianfetamina (MDA) & 10,97 & 0,899 \\
Femproporex & 11,97 & 0,981 \\
3,4-Metilenodioxietilanfetamina (MDEA) & 12,63 & 1,035 \\
Cafeína & 13,22 & 1,084 \\
Difenidramina & 13,54 & 1,109 \\
Clorfeniramina & 14,99 & 1,228 \\
\hline
\end{tabular}

TABELA III - Resultados das análises de amostras de urina de voluntários, seu relato de uso e modalidades esportivas que praticam

\begin{tabular}{lcccccc}
\hline Voluntário & Relato de uso & Modalidade esportiva & \multicolumn{4}{c}{ Resultado $(\mu \mathrm{g} / \mathrm{mL})$} \\
& & & EPH & PEPH & NEPH & MEPH \\
\hline A & SCE (eventual) & corrida/natação & 14,9 & 0,9 & 3,3 & - \\
B & SCE (1x/dia) & musculação & 8,3 & 1,2 & 1,5 & - \\
C & SCE (5x/sem) & musculação/jiu-jitsu & 3,6 & - & 1,8 & - \\
D & SCE (1x/dia) & musculação/jiu-jitsu & 32,6 & 1,7 & 2,9 & - \\
E & SCE (1x/dia) & musculação/jiu-jitsu & 17,6 & 1,7 & 1,9 & - \\
F & SCE (1x/dia) & musculação/jiu-jitsu & 11,3 & 2,0 & 3,2 & - \\
G & S (NR) & musculação & 2,0 & - & - & - \\
H & EPH (3x/dia) & NR & - & 4,8 & 94,0 & - \\
I & & NR & - & 14,0 & 118,3 & 3,8 \\
J & EFE (1x/dia) & NR & - & 209,8 & 7,5 & - \\
\hline
\end{tabular}

EPH: efedrina, PEPH: pseudoefedrina, NEPH: norefedrina, MEPH: metilefedrina, SCE: suplemento nutricional contendo efedrina, S: suplemento nutricional não especificado, EFE: especialidade farmacêutica contendo pseudoefedrina, NR: não relatado, sem: semana.

nificativa dos casos positivos de dopagem. Segundo levantamento estatístico realizado em 1999 pelo Comitê Olímpico Internacional, do total de casos positivos para agentes estimulantes, $70 \%$ foram para efedrinas sendo a mais freqüente a pseudoefedrina (Gmeiner et al., 2002).

Até o presente momento, a maneira mais eficaz de minimizar a dopagem nos esportes tem sido seu controle sistemático, através da realização de análises toxicológicas em material biológico fornecido pelo atleta. Com relação às efedrinas, diversos métodos têm sido relatados na literatura científica para sua detecção em urina utilizando as técnicas de cromatografia líquida de alta efici- ência (Gmeiner et al, 2002), cromatografia em fase gasosa (Van Eenoo, 2001; ), eletroforese capilar (MateusAvois, Mangin, Saygy, 2003) e espectrometria de massas (Spyridaki, 2001; Van der Merwe, Hendrikz, 1995; Tseng, 2003). A maioria destes métodos cromatográficos reporta extensas fases de preparação da amostra ou não permitem eficiente separação de efedrina e pseudoefedrina (Van der Merwe, Hendrikz, 1995; Van Eenoo, 2001; Spyridaki, 2001; Tseng, 2003).

No presente trabalho, a cromatografia em fase gasosa com detector de nitrogênio/fósforo foi utilizada como técnica para quantificação de efedrina, pseudoefedrina, 
norefedrina e metilefedrina em urina. O método, no qual se utiliza extração líquido-líquido com éter terc-butilmetílico e posterior derivação do extrato com anidrido trifluoroacético, demonstrou ser rápido, prático e eficiente. Cerca de 50 amostras de urina podem ser preparadas e analisadas por um único analista em um período de 4 horas. Apesar de consistir de uma única fase de extração, apresentou bons índices de recuperação para todos os fármacos pesquisados (recuperação > 67\%). Ácido acético glacial foi acrescentado ao extrato para que as aminas fossem convertidas à sua forma ionizada e desta forma evitar perdas dos analitos advindas da evaporação, etapa crítica do processo de extração das efedrinas (Spyridaki, 2001).

A reação com o anidrido trifluoroacético deu origem a derivados com boa resolução cromatográfica, possibilitando a separação dos isômeros efedrina e pseudoefedrina, o que não foi observado em outros métodos descritos na literatura (Van Eenoo, 2001; Spyridaki, 2001; Tseng, 2003). Entretanto, o derivado trifloroacetil da metilefedrina demonstrou ser instável, retornando a sua estrutura original em poucas horas. Isto pode ser constatado pela presença inicial no cromatograma de um pico em 5,92 minutos, correspondente ao derivado trifluoroacetil, que ia diminuindo de intensidade conforme aumentava seu pico de determinação em 8,07 minutos, correspondente a metilefedrina não derivada. Este fato também foi observado através de injeções consecutivas de diversas alíquotas do extrato derivado de metilefedrina, em um equipamento de espectrometria de massas associada à cromatografia em fase gasosa, ao longo de 2 horas. Durante esse período observou-se a reversão total do derivado trifluoroacetil em metilefedrina, em uma hora. Devido a este fato, após a etapa de derivação o extrato deve permanecer em repouso pelo menos uma hora antes de iniciar as injeções no equipamento de cromatografia.

O método apresentou boa linearidade nas faixas de concentração estudadas. Poucas variações foram observadas, principalmente nos estudos de precisão intraensaio $(\mathrm{CV}<9 \%)$ para todos os analitos pesquisados. Boa sensibilidade também foi obtida utilizando somente $0,2 \mathrm{~mL}$ de amostra de urina.

O estudo de especificidade demonstrou que as aminas simpatomiméticas e os fármacos pesquisados neste ensaio não interferiram na detecção e quantificação das efedrinas. Entretanto, para identificação inequívoca da substância presente na amostra, a espectrometria de massas associada à cromatografia em fase gasosa (CG/EM) deve ser utilizada, o que pode ser feito pela injeção de uma alíquota do extrato obtido pelo método proposto no equipamento de CG/EM.

Sete das dez amostras provenientes de voluntários que relataram uso de suplementos ou especialidades farmacêuticas contendo efedrinas apresentaram concentrações acima dos limites estabelecidos pelo COI segundo a lista de substâncias proibidas de 2003. Apenas um voluntário apresentou nível quantificável de metilefedrina.

\section{CONCLUSÃO}

O método estudado e validado para determinação de efedrina, pseudoefedrina, metilefedrina e norefedrina por cromatografia em fase gasosa com detector de nitrogênio/ fósforo demonstrou ser prático, rápido e eficiente, podendo ser aplicado no controle da dopagem no esporte.

\section{ABSTRACT}

\section{Gas chromatographic method for the determination of ephedrines in urine for doping control purposes}

Ephedrines are sympathomimetic amines present in many pharmaceutical preparations used in the treatment of respiratory diseases due to their actions against broncospasm and congestion. Nowadays, several products sold as nutritional supplements contain ephedrines and are widely used in a diverse range of sports as weight loss aids and enhancement of athletic performance. However, the abuse of ephedrines may lead to a number of adverse effects including hypertension, headache, tachycardia and seizure. Due to their CNS stimulating action, ephedrines are included in the prohibited list of substances by the International Olympic Committee (IOC). Urinary threshold concentrations were established (ephedrine and methylephedrine $>10 \mu \mathrm{g} / \mathrm{mL}$ ). The aim of this work was to develop and validate a method for the simultaneous determination of ephedrines present in urine samples for doping purposes by gas chromatography with nitrogenousphosphorous detector (GC/NPD). The method was based on a liquid-liquid extraction of alkalinized urine and further derivatization with trifluoroacetic anhydride (TFA). The validated method showed to be simple, practical and linear at the studied concentrations range. Urine samples collected from volunteers, who reported the use of ephedrines, were submitted to the proposed method.

UNITERMS: Ephedrines. Doping in sports. Gas chromatography.

\section{REFERÊNCIAS BIBLIOGRÁFICAS}

BENT, S.; TIEDT, T. N.; ODDEN, M. C.; SHLIPAK, M. G. The relative safety of ephedra compared with other herbal products. Ann. Intern. Med., Philadelphia, v.138, p.46871, 2003. 
BERLIN, I.; WAROT, D.; AYMARD, G.; ACQUAVIVA, E.; LEGRAND, M.; LABARTHE, B.; PEYRONT, I.; DIQUET, B.; LECHAT, P. Pharmacodynamics and pharmacokinetics of single nasal $(5 \mathrm{mg}$ and $10 \mathrm{mg}$ ) and oral $(50 \mathrm{mg})$ doses of ephedrine in healthy subjects. Eur. J. Clin. Pharmacol., Berlin, v.57, p.447-455, 2001.

BOHN, A. M.; KHODAEE, M.; SCHWENK, T. L. Ephedrine and other stimulants as ergogenic aids. Curr. Sports Med. Rep., Philadelphia, v.2, p.220-225, 2003.

BRASIL, Agência Nacional de Vigilância Sanitária, Resolução - RDC n ${ }^{\circ}$ 96, de 8 de novembro de 2000. Diário Oficial da União, Brasília, 10 de novembro de 2000 .

CATLIN, D. H.; MURRAY, T. H. Performance-enhancing drugs, fair competition, and Olympic sport. JAMA, Chicago, v.276, n.3, p.231-7, 1996.

DELBEKE, F.T.; VAN EENOO, P.; VAN THUYNE, W.; DESMET, N. Prohormones and sport. J. Steroid. Biochem. Mol. Biol., Oxford, v. 1804, p. 1-7, 2003.

GMEINER, G.; GEISENDORF, T.; KAINZBAUER, J.; NIKOLAJEVIC, M.; TAUSCH, H. Quantification of ephedrines in urine by column-switching highperformance liquid chromatography. J Chromatogr. B, Amsterdam, v. 768, p. 215-221, 2002.

GURLEY, B. J.; GARDNER, S. F.; HUBBARBD, M. A. Content versus label claims in ephedra-containing dietary supplements. Am. J. Health Syst. Pharm., Bethesda, v.57, p.963-969, 2000.

INTERNATIONAL OLYMPIC COMMITTEE. List of prohibited classes of substances and prohibited methods. Lausanne, 2003.

INTERNATIONAL OLYMPIC COMMITTEE. List of prohibited classes of substances and prohibited methods. Lausanne, 2004.

JUHN, M. S. Popular sports supplements and ergogenic aids. Sports Med., Auckland, v.33, n.12, p.921-939, 2003.
LIEBERMAN, H. R. The effects of ginseng, ephedrine and caffeine on cognitive performance, mood and energy. Nutr. Rev., Baltimore, v.59, n.4, p.91-102, 2001.

MATEUS-AVOIS, L.; MANGIN, P.; SAYGY, M. Development and validation of a capillary zone electrophoresis method for the determination of ephedrine and related compounds in urine without extraction. $J$ Chromatogr. B, Amsterdam, v. 791, p. 203-216, 2003.

SILVA, O.A.; YONAMINE, M. Aspectos farmacológicos da dopagem no esporte. In: AMATUZZI, M.M., CARAZZATO, J.G., eds. Medicina do esporte. São Paulo: Ed. Roca, 2004, p.141-157.

SPYRIDAKI, M-H. E.; TSITSIMPIKOU, C. J.; SISKOS, P. A.; GEORGAKOPOLOUS, C.G. Determination of ephedrines in urine by gas chromatography - mass spectrometry. J. Chromatogr. B, Amsterdam, v.758, p.311-314, 2001.

TSENG, Y. L.; HSU, H. R.; KUO, F. H.; SHIEH, M. H.; CHANG, C. F. Ephedrines in over-the-counter cold medicines and urine specimens collected during sport competitions. J. Anal. Toxicol., Niles, v.27, n.6, p.359$365,2003$.

VAN DER MERWE, P. J.; HENDRIKZ, S. E. Identification of ephedrines as their carbon disulfide derivatives. $J$. Chromatogr. B, Amsterdam, v.663, p.160-166, 1995.

VAN EENOO, P.; DELBEKE, F. T.; ROELS, K.; DE BACKER, P. Simultaneous quantitation of ephedrines in urine by gas chromatography - nitrogen - phosphorus detection for doping control purposes. J. Chromatogr. B., Amsterdam, v.760, p.255-261, 2001.

VANSAL, S. S.; FELLER, D. R. Direct effects of ephedrine isomers on human $\beta$-adrenergic receptor subtypes. Biochem. Pharmacol., Oxford, v.58, p.807-810, 1999.

YONAMINE, M.; GARCIA, P.R.; MOREAU, R.L.M. Nonintentional doping in sports. Sports Med., Auckland, v.34, n. 11, p.697-704, 2004.

Recebido para publicação em 19 de abril de 2004. Aceito para publicação em 04 de novembro de 2004. 